\title{
SIMPLICITY OF CERTAIN GROUPS OF DIFFEOMORPHISMS
}

\author{
BY JOHN N. MATHER ${ }^{1}$
}

Communicated by Shlomo Sternberg, October 1, 1973

D. Epstein has shown [1] that for quite general groups of homeomorphisms, the commutator subgroup is simple. In particular, let $M$ be a manifold. (In this note, we assume all manifolds are finite dimensional, Hausdorff, class $C^{\infty}$, and have a countable basis for their topology.) By a $C^{r+}$ mapping (resp. diffeomorphism) we mean a $C^{r}$ mapping (resp. diffeomorphism) whose $r$ th derivative is Lipschitz. Let $\operatorname{Diff}(M, r)$ (resp. $\operatorname{Diff}(M, r+))$ denote the group of $C^{r}$ (resp. $C^{r+}$ ) diffeomorphisms $h$ of $M$ such that there is an isotropy $H_{t}$ of $h$ to the identity, and a compact set $K$ such that $H_{t}(x)=x$ if $x \in M-K$. Epstein showed in [1] that the commutator subgroup of $\operatorname{Diff}(M, r)$ (resp. $\operatorname{Diff}(M, r+)$ ) is simple. Thurston announced in [4] that $\operatorname{Diff}(M, \infty)$ is simple. Let $n=\operatorname{dim} M$. In this note we announce the following two results.

THEOREM 1. If $\infty \geqq r \geqq n+1$, then $\operatorname{Diff}(M, r+)$ is simple.

THEOREM 2. If $\infty \geqq r \geqq n+1$, then $F \Gamma_{n}^{r+}$ is $(n+1)$-connected.

Here $F \Gamma_{n}^{r+}$ denotes Haefliger's classifying space for codimension $n$ foliations of class $C^{r+}$. These two theorems are closely related by results of Thurston (cf. [2], [4]). The case $r=\infty$ of these theorems is due to Thurston [4].

Here we outline a proof of Theorem 1. By Epstein's theorem, it is enough to show $\operatorname{Diff}(M, r+)$ is equal to its own commutator subgroup. A well-known argument shows that it is enough to prove the latter in the case $M=\boldsymbol{R}^{n}$. Let $A>1$. Let $D_{0}=\left\{x \in \boldsymbol{R}^{n}:-2 \leqq x_{j} \leqq 2,1 \leqq j \leqq n\right\}$. For $1 \leqq i \leqq n$, let

$$
D_{i}=\left\{x \in \boldsymbol{R}^{n}:-2 \leqq x_{j} \leqq 2,1 \leqq j<i,-2 A \leqq x_{j} \leqq 2 A, i \leqq j \leqq n\right\} .
$$

Let $\alpha \in \operatorname{Diff}\left(\boldsymbol{R}^{n}, \infty\right)$ be such that $\alpha(x)=A x$ if $x \in D_{0}$. Let $\rho$ be a $C^{\infty}$ real valued function on $\boldsymbol{R}^{n}$, with compact support, such that $0 \leqq \rho \leqq 1$, and $\rho=1$ on $D_{1}$. Let $\tau_{i}=\exp \left(\rho \partial / \partial x_{i}\right)$. Then $\tau_{i} \in \operatorname{Diff}\left(\boldsymbol{R}^{n}, \infty\right), 1 \leqq i \leqq n$.

AMS (MOS) subject classifications (1970). Primary 58D05; Secondary 57D30.

${ }^{1}$ This research was partially supported by NSF Grant GP-31359X-1 and a Sloan Foundation Fellowship. 
Lemma. There exists $A_{0}$ such that the following holds. Let $A \geqq A_{0}$. Let $f$ be a $C^{r+}$ diffeomorphism of $\boldsymbol{R}^{n}$, with support in $D_{0}$, and sufficiently close to the identity (with respect to the $C^{r+}$ topology). Suppose $\infty>r \geqq n+1$. Then there exist $g_{0}, g_{1}, \cdots, g_{n}, \lambda_{1}, \cdots, \lambda_{n} \in \operatorname{Diff}\left(R^{n}, r+\right)$ such that

$$
\begin{aligned}
\alpha f g_{n} \alpha^{-1} & =g_{0}, \\
\lambda_{i} g_{i-1} \tau_{i} \lambda_{i}^{-1} & =g_{i} \tau_{i}, \quad 1 \leqq i \leqq n .
\end{aligned}
$$

Proof that The Lemma IMPles Theorem 1. It is enough to show that any diffeomorphism such as $f$ is a product of commutators, since any element of $\operatorname{Diff}\left(\boldsymbol{R}^{n}, r+\right)$ is a product of conjugates of such diffeomorphisms. Now if $u \in \operatorname{Diff}\left(\boldsymbol{R}^{n}, r+\right)$, let $[u]$ denote its image in the commutator quotient group. From $(1)$, we get $[f]\left[g_{n}\right]=\left[g_{0}\right]$, and from $\left(2_{i}\right)$, we get $\left[g_{i-1}\right]=\left[g_{i}\right], 1 \leqq i \leqq n$. Hence $[f]=1$. Q.E.D.

OUTLINE OF THE PROOF OF THE LEMMA IN THE CASE $r<\infty$. Let $B_{\delta}$ denote the subset of $\operatorname{Diff}\left(\boldsymbol{R}^{n}, r+\right)$ consisting of $g$ with support in $D_{0}$ such that

$$
\sup _{x \neq y}\left\|D^{r} g(x)-D^{r} g(y)\right\| /\|x-y\|<\delta .
$$

For $\delta>0$ sufficiently small, and $f$ sufficiently near the identity, there exists a mapping $\Phi: B_{\delta} \rightarrow B_{\delta}$ such that if $g \in B_{\delta}$ and $g_{n}=\Phi(g)$, then there exist $g_{1}, \cdots, g_{n}, \lambda_{1}, \cdots, \lambda_{n} \in \operatorname{Diff}\left(\boldsymbol{R}^{n}, r+\right)$ such that

$$
\begin{aligned}
\alpha f g \alpha^{-1} & =g_{0}, \\
\lambda_{i} g_{i-1} \tau_{i} \lambda_{i}^{-1} & =g_{i} \tau_{i}, \quad 1 \leqq i \leqq n .
\end{aligned}
$$

The mapping $\Phi$ is continuous with respect to the $C^{r}$ topology. Since $B_{\delta}$ is compact with respect to the $C^{r}$ topology, and convex, it follows from the Schauder-Tychonoff fixed point theorem that $\Phi$ has a fixed point. But such a fixed point provides a solution of the equations (1), $\left(2_{\mathbf{i}}\right)$.

We can only sketch the idea of the construction of $\Phi$. If $u: \boldsymbol{R}^{n} \rightarrow \boldsymbol{R}^{n}$ vanishes outside a compact set, we define

$$
\|u\|_{r+}=\sup _{x+y}\left\|D^{r} u(x)-D^{r} u(y)\right\| /\|x-y\| .
$$

Then it is easy to see that if $g_{0}$ is defined by (3), we have

$$
\left\|g_{0}-\mathrm{id}\right\|_{r+}<A^{-r}\|f g-\mathrm{id}\|_{r+}
$$

Then we construct $g_{1}, \cdots, g_{n}$ inductively. Supposing $g_{i-1}$ has been defined, has support in $D_{i}$, and is near the identity, we construct $g_{i}$ to have support in $D_{i+1}$, to be near the identity and to satisfy

$$
\left\|g_{i}-\mathrm{id}\right\|_{r+} \leqq C A\left\|g_{i-1}-\mathrm{id}\right\|_{r+} .
$$


Here, $C$ is a constant independent of $A$. By taking $A$ sufficiently large, and $f$ sufficiently near the identity in relation to $\delta$, we have that $\Phi$ maps $B_{\delta}$ into itself, where we define $\Phi(g)=g_{n}$.

\section{REFERENCES}

1. D. B. A. Epstein, The simplicity of certain groups of homeomorphisms, Compositio Math. 22 (1970), 165-173. MR 42 \#2491.

2. J. Mather, Loops and foliations, Proc. Internat. Conf. on Manifolds, Tokyo, 1973, (to appear).

3. - Commutators diffeomorphisms, Comment. Math. (to appear).

4. W. Thurston, Foliations and groups of diffeomorphisms, Bull. Amer. Math. Soc. (to appear).

Department of Mathematics, Harvard University, Cambridge, Massachusetts 02138 